\title{
Estado actual de las investigaciones y riesgos del uso de los cigarrillos electrónicos
}

\author{
Current status of investigations and risks of the use of electronic cigarettes
}

\section{Sr. Editor:}

El cigarrillo electrónico (E-cig) o vapeador, desde su creación a inicios de la década de 2000 por el farmacéutico chino Hon Lik, ha sido considerado eficaz para evitar los riesgos atribuidos a la combustión del tabaco (1). De hecho, fumar cigarrillos es la causa principal de muchos tipos de cáncer (2), destacando el de pulmón (3). Por ello, en la $56^{\circ}$ Asamblea Mundial de la Salud, de mayo de 2003, se adoptó el Convenio Marco de la Organización Mundial de la Salud para el Control del Tabaco (CMCT). El Perú suscribió el CMCT el 21 de abril de 2004, aprobándolo el Congreso de la República el 16 de julio de 2004 (4). Dos años más tarde, se promulgaría la Ley General para la prevención y control de los riesgos del consumo del tabaco (5).

Sin embargo, los estudios que afirman la inocuidad de los cigarrillos electrónicos sobredimensionan sus beneficios. Así, Nutt et al. (6,7), afirman que los cigarrillos electrónicos son $95 \%$ más seguros que los cigarrillos convencionales, mientras que Bullen y col. (8), subestiman los efectos de la terapia de reemplazo de nicotina (TNR) (8). Del mismo modo, la Public Health England, la Royal College of Physicians, la Royal Society for Public Health y la National Health Service de Reino Unido sostienen que el cigarrillo electrónico contribuye a reducir el hábito de fumar.

Por el contrario, Kalkhoran y Glantz (9) señalan que las probabilidades de abandonar el hábito de fumar cigarrillos es un $28 \%$ más baja entre los que usaron cigarrillos electrónicos respecto de aquellos que no lo hicieron. En igual sentido, Glantz y Bareham (10), advierten que "los cigarrillos electrónicos estimulan la adicción a la nicotina y expanden el mercado para consumidores cada vez más jóvenes", incluso de otras drogas como la marihuana (11). Coincidentemente, el estudio de Kulik, Ling y Glantz (12) en veintiocho países de la Unión Europea encontró que el uso de cigarrillos electrónicos se vincula a la inhibición del consumo de cigarrillos convencionales y no al abandono del hábito de fumar.

Los resultados del año 2017 de la encuesta sobre alcohol, el tabaco y otras drogas en España (EDADES), mostraron que el grupo etario de jóvenes fumadores, entre los 15 a 34 años, poseía relativa facilidad para acceder al tabaco, usaba dispositivos electrónicos de nicotina, al tiempo que también alternaban el consumo de tabaco con el cannabis (13). Resultados similares permiten al U.S. Departament of Health \& Human Services hablar de una epidemia entre los jóvenes, caracterizada por el aumento en las dosis consumidas de nicotina y el temor que ello derive en adicción, favorecido por la mayor presencia de E-cig en las escuelas (14).

En nuestro país la ley contra el tabaco ha devenido en obsoleta por el avance tecnológico de los cigarrillos electrónicos, a pesar de la preocupación del Ministerio de Salud (15). Por ello, siguiendo el principio precautorio desarrollado por nuestro Tribunal Constitucional (16), y ante los indicios de amenaza a la salud pública, es necesario regular la comercialización, publicidad y uso de los cigarrillos electrónicos y afines. Del mismo modo, debería constituir una prioridad del Ministerio de Salud el financiamiento de investigaciones nacionales, en los establecimientos públicos a su cargo, sobre los nuevos hábitos y tecnologías disponibles para el consumo de

1. Grupo de Investigación Diseñando el Perú: Estado, ciudadanía, intelectuales y política, Facultad de Ciencias Sociales, Universidad Nacional Mayor de San Marcos. Lima, Perú.

a. Bachiller en Derecho y Sociología 
nicotina, a fin de comprender los efectos al mediano y largo plazo en la salud, tal como lo recomiendan Green, Fielding y Brownson (17).

\section{Frank Rojas-Alarcón 1,a}

\section{Correspondencia:}

Frank Rojas-Alarcón

Calle Matamoros s/n, Fundo Los Laras, Pachacámac, Lima, Perú.

Teléfono: 51992243985

ORCID: 0000-0002-1223-7262

Correo electrónico: frank.rojas@unmsm.edu.pe

\section{REFERENCIAS BIBLIOGRÁFICAS}

1. Grana R, Benowitzy N, Glantz SA. E-Cigarettes: A Scientific Review. Circulation. 2014; 129:1972-1986. doi:10.1161/CIRCULATIONAHA.114.007667

2. Cogliano VJ, Baan R, Staif K. Preventable Exposures Associated With Human Cancers. J. Natl. Cancer Inst. 2011; 103(24):1827-1839. doi: 10.1093/jnci/ djr483

3. Wipfli H, Samet JM. One Hundred Years in the Making: The Global Tobacco Epidemic. Annu. Rev. Public Health. 2016; 37:149-166. doi: https:// doi.org/10.1146/annurev-publhealth-032315-021850

4. Congreso de la República. Resolución Legislativa 28280 que aprueba el Convenio Marco de la OMS para el control del tabaco. Diario El Peruano. 2004 julio 17.

5. Congreso de la República. Ley 28705 para la prevención y control de los riesgos del consumo del tabaco. Diario El Peruano. 2006 abril 06.

6. Nutt DJ, Phillips LD, Balfour D, et al. Estimating the Harms of Nicotine-Containing Products Using the MCDA Approach. Eur Addict Res. 2014; 20:218225. doi: $10.1159 / 000360220$

7. Nutt DJ, Phillips LD, Balfour D, et al. E-cigarettes are less harmful than smoking. Lancet. 2016; 387:1160-1162. doi: https://doi.org/10.1016/S01406736(15)00253-6

8. Bullen C, Howe C, Laugesen M, et al. Electronic cigarettes for smoking cessation: a randomised controlled trial. Lancet. 2013; 382(9905):1629-1637. doi: http://dx.doi.org/10.1016/S0140-6736(13)61842-5

9. Kalkhoran S, Glantz SA. E-cigarettes and smoking cessation in real-world and clinical settings: a systematic review and meta-analysis. Lancet. 2016; 4(2):116-128. doi: https://doi.org/10.1016/S22132600(15)00521-4

10. Glantz SA, Bareham DW. E-Cigarettes: Use, Effects on Smoking, Risks, and Policy Implications. Annu. Rev. Public Health. 2018; 39(1):215-235. doi: https:// doi.org/10.1146/annurev-publhealth-040617-013757

11. National Academies of Sciences, Engineering, and Medicine. Public Health Consequences of E-Cigarrettes. Washington DC: The National Academies Press. 2018. doi: https://doi. org $/ 10.17226 / 24952$

12. Kulik MC, Lisha NE, Glantz SA. E-cigarettes associated with depressed smoking cessation: a cross-sectional study of 28 European Union Countries. Am J Prev Med. 2018; 54 (4):603-609. doi: $10.1016 /$ j.amepre.2017.12.017

13. Rábade Castedo C, Granda-Orive JI, GonzálesBarcala FJ. Incremento de la prevalencia del tabaquismo: ¿causas y actuación? Arch. Bronconeumol. 2019; 55:557-558. doi: https://doi. org/10.1016/j.arbres.2019.06.021

14. U.S. Departament of Health \& Human Services. Surgeon General releases advisory on E-cigarette epidemic among youth. Washington D.C, 2018. (citado el 25 diciembre 2019). Disponible en: https:// www.hhs.gov/about/news/2018/12/18/surgeongeneral-releases-advisory-e-cigarette-epidemicamong-youth.html

15. Tribunal Constitucional. Expediente 3510-2003AA/TC. Lima. 2005 abril 13. (citado el 25 diciembre 2019). Disponible en: https://www.tc.gob. pe/jurisprudencia/2005/03510-2003-AA.html

16. Ministerio de Salud del Perú. El cigarrillo electrónico incrementa cuatro veces más la adicción al tabaco convencional. Lima: Ministerio de Salud del Perú; 2019. (citado el 10 agosto 2019). Disponible en: https://www.gob.pe/institucion/minsa/ noticias/28917-el-cigarrillo-electronico-incrementacuatro-veces-mas-la-adiccion-al-tabacoconvencional

17. Green LW, Fielding JE, Brownson RC. The Debate about Electronic Cigarettes: Harm Minimization or the Precautionary Principle. Annu Rev Public Health. 2018; 39(1):189-191. doi: https://doi.org/10.1146/ annurev-publhealth-102417-124810

Recibido: 12/08/2019 Aceptado: 07/01/2020 